\title{
Efectos de la intensificación agrícola sobre la estructura y diversidad del paisaje en la región sojera de Uruguay
}

\author{
Guadalupe Tiscornia ${ }^{1, \bigotimes} ;{\text { Marcel } \text { AchKar }^{2} \& \text { Alejandro Brazeiro }}^{3}$ \\ 1.Unidad de Agroclima y Sistemas de Información (GRAS), INIA, Canelones, Uruguay. 2. LDSGAT, IECA, Facultad \\ de Ciencias, Universidad de la República, Montevideo, Uruguay. 3. BEC, IECA, Facultad de Ciencias, Universidad de la \\ República, Montevideo, Uruguay.
}

\begin{abstract}
Resumen. En las últimas décadas, impulsado por la creciente demanda de alimentos y biocombustibles a nivel mundial, la actividad agrícola ha sufrido un crecimiento exponencial a expensas de ecosistemas naturales. Uruguay no es ajeno a este fenómeno. Este avance ha ocurrido con mayor intensidad en el litoral oeste del país, impulsado en la última década por el cultivo de soja. El objetivo del trabajo fue evaluar los cambios territoriales ocurridos a nivel de paisaje, asociados al proceso de intensificación agrícola en un departamento (división política) del litoral oeste de Uruguay, en el período 1990-2009. Las tierras destinadas a cultivos aumentaron $27 \%$, a expensas de la disminución de la superficie de bosque nativo (BN) y pastizales o campo natural (CN). El BN disminuyó 30\%, y se perdieron principalmente los fragmentos $<10$ ha. El CN se redujo $23 \%$, y desaparecieron principalmente fragmentos de hasta 50 ha. La conectividad (distancia promedio de separación entre parches) en el caso de CN pasó de $168 \mathrm{~m}$ a $192 \mathrm{~m}$, mientras que para el BN fue $365 \mathrm{~m}$ en 1990 y 255 m en 2009. Esto último concuerda con una distribución más dispersa en 1990, que se haría más compacta en 2009 por la desaparición de fragmentos pequeños en sectores periféricos. En relación al grado de fragmentación, para el CN aumentó de forma leve, mientras que para el BN disminuyó; esto podría estar causado por la desaparición de fragmentos pequeños (<10 ha). Entre los años 1990 y 2009, desaparecieron 2000 fragmentos de $\mathrm{BN}$ menores a 10 ha y 2500 fragmentos de CN entre 1 y 100 ha. En la mayoría de los casos se transformaron en cultivos de soja. Los efectos de este proceso en la producción agrícola están cuantificados, pero sus repercusiones sobre la capacidad del paisaje de sostener su biodiversidad y brindar una amplia gama de bienes y servicios ecosistémicos, son aun poco conocidas.
\end{abstract}

[Palabras clave: biodiversidad, cambio de uso de suelo, fragmentación, pérdida de hábitat]

\begin{abstract}
AвSTRACT. Effects of agricultural intensification on landscape structure and diversity on the Uruguayan soybean region: In recent decades, driven by the worldwide growing demand for food and biofuels, agriculture has grown exponentially at the expense of natural ecosystems. The same phenomenon is happening in Uruguay, with greater intensity on the west region of the country during the last decade, driven by the soybean crop. The objective of our study was to assess the territorial changes occurred at the landscape level, associated with agricultural intensification, on a department (political division) on the western region of Uruguay, between 1990 and 2009. Crop areas increased $27 \%$ during this period, advancing over native forest (BN) and grasslands / rangelands $(\mathrm{CN})$ areas. The $\mathrm{BN}$ decreased $30 \%$ mainly through the loss of small fragments $(<10 \mathrm{ha})$. The $\mathrm{CN}$ was reduced by a $23 \%$, mostly through the disappearance of fragments up to 50 ha. In relation to connectivity, average distances between patches of $\mathrm{CN}$ have changed from $168 \mathrm{~m}$ to $192 \mathrm{~m}$, whereas in BN they have gone from $365 \mathrm{~m}$ to $255 \mathrm{~m}$ in 2009. This last observation is consistent with a more disperse distribution in 1990, that will become more compact by 2009 through the disappearance of small peripheral patches. The fragmentation of $\mathrm{CN}$ showed a slightly increment in the period, while a decrease was observed in BN, possibly caused by the disappearance of small fragments (less than 10 ha). Between 1990 and 2009, 2000 fragments of BN <10 ha and 2500 fragments from 1-100 CN have disappeared, becoming in most cases on soybean crops. The effects of this process on agricultural production has been quantified but the impact on landscape's capacity to sustain biodiversity and provide a wide range of ecosystem goods and services, is still poorly understood.
\end{abstract}

[Keywords: biodiversity, land-use change, fragmentation, habitat loss]

\section{INTRODUCCIÓN}

La expansión agrícola es uno de los principales factores de cambios en el tipo de aprovechamiento que se realiza en los distintos ecosistemas terrestres (uso del suelo). Esta expansión es una parte importante del "cambio global" (Green et al. 2005). Influye sobre el clima, ciclos del agua, carbono y nitrógeno de la biósfera, emisiones de gases

Editor Asociado: Esteban Jobbagy

$\bowtie$ gtiscornia@inia.org.uy causantes del efecto invernadero y sobre la biodiversidad (Foley et al. 2005; Paruelo et al. 2006). Ante la demanda creciente de productos de origen agrícola, este proceso parece irreversible. Sin embargo, y a pesar de su probable beneficio económico, las consecuencias sociales y ambientales que trae aparejadas advierten acerca de la importancia de planificar y regular la expansión del área cultivada (MEA 2005; Paruelo et al. 2006). 
Por su parte, nuestra región no es ajena a este fenómeno de expansión agrícola. Existen varios trabajos que han analizado los cambios territoriales ocurridos en la zona (Uruguay, Argentina, Paraguay y Brasil). Allí se evidencia una expansión de los cultivos, en particular la soja (Huang et al. 2009; Schlesinger 2008; Huang et al. 2007; Paruelo et al. 2006; Guerschman 2005). En el caso puntual de Uruguay, el sector agropecuario viene atravesado en la última década un proceso de transformación, con cambios importantes en la base productiva y social. El sector agrícola en Uruguay se desarrolló con el objetivo de satisfacer las demandas del mercado interno, y alcanzó una superficie máxima de ocupación del suelo a principios de la década de 1950 con casi 1000000 ha. Progresivamente, y a consecuencia del abandono del modelo de sustitución de importaciones, la actividad agrícola comienza a reducir las áreas cultivadas y el número de agricultores. Presenta un proceso continuo de tecnificación, aumento de la producción y concentración de la producción (De los Campos et al. 2002). En la década de 1990 llega a un promedio de 470000 ha, ocupadas principalmente con arroz, trigo, cebada, girasol, maíz y sorgo. Esta superficie genera cerca de 50\% del VBP del sector agropecuario (Achkar et al. 2000; Arbeletche \& Carballo 2006). A principios de siglo XXI, la irrupción del cultivo de soja como expansión de la frontera agrícola argentina desde el litoral oeste del país convierte a este producto en el principal rubro de la agricultura nacional (Figura 1). Este proceso desplaza a la producción de arroz, que durante muchos años constituyó el generador principal de excedentes exportables del sector agrícola nacional; tuvieron lugar transformaciones territoriales a causa de la competencia por tierra entre la producción agrícola ganadera convencional, el cultivo de soja y oleaginosas y la forestación (Achkar et al. 2008; Brazeiro et al. 2008). Las plantaciones, en particular en el litoral oeste del Uruguay, se desarrollan en Soriano y Río Negro, vinculadas a una oferta de tecnología compuesta de semillas transgénicas resistentes al glifosato y paquetes de siembra directa. En el contexto de un mercado internacional relativamente sostenido se generó un escenario positivo gracias a un paquete tecnológico que asegura una productividad media entre 1500 y 2000 $\mathrm{kg} / \mathrm{ha}$ y al libre ingreso de capitales al sector. Esto despertó el interés de grandes inversores (muchos de ellos extranjeros), con lógicas de producción muy diferentes a la del agricultor "tradicional" (Arbeletche \& Carballo 2006). Cabe destacar también que este proceso viene acelerando las tendencias históricas de concentración de la producción y desplazamiento de productores familiares. En esta zona y debido al avance territorial de los cultivos de soja, estos productores han disminuido en cantidad de $15 \%$ en 2000 a 6\% en 2009 (Oyhancabal \& Narbondo 2010).

Esta expansión agrícola también genera aumentos en la fragmentación del paisaje en los espacios rurales. La fragmentación es un proceso dinámico por el cual un determinado ambiente va quedando reducido a fragmentos, parches o islas de menor tamaño, más o menos conectadas entre sí, en una matriz diferente de la original (Forman 1995). En este contexto se supone que la fragmentación siempre está asociada a acciones antrópicas, que conllevan una modificación del territorio, que se traduce en una pérdida importante de hábitats naturales. Por lo general, conduce a disminuciones poblacionales e incluso a extinciones de especies (Fahrig 2001; Múgica de la Guerra et al. 2002). El tamaño y la forma de los fragmentos condicionan en gran medida las posibilidades de mantener ciertas poblaciones. Al aumentar la fragmentación se producirían aumentos en la probabilidad de

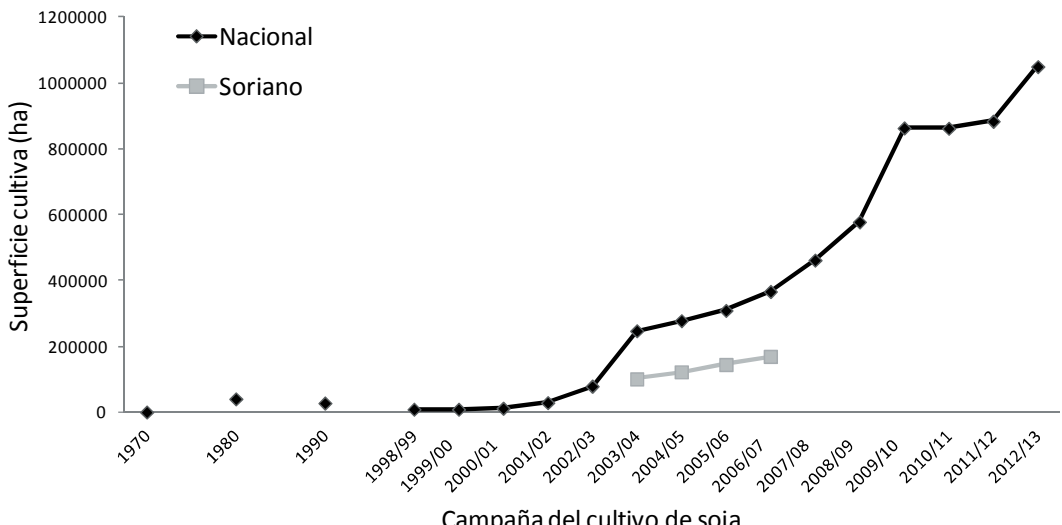

Campaña del cultivo de soja
Figura 1. Superficie de soja cultivada (ha) en Uruguay (negro) y en Soriano (gris), en el período 1970-2010. Notar que la información de Soriano solo se dispone para el período 2003-2007. Datos tomados de MGAP-DIEA.

Figure 1. Planted soybean area (ha) in at Uruguay (black) and in Soriano (gray) during 1970-2010. Note that information for Soriano is only available from 2003 to 2007. From MGAP-DIEA. 
extinción local de especies de fauna autóctona. A medida que el tamaño de los fragmentos se reduce y el aislamiento crece, numerosas especies se hacen más vulnerables al efecto borde (depredación de nidos, invasiones, enfermedades, etc.) y pasados ciertos umbrales, la extinción se hace inevitable (Fahrig 2001; Múgica de la Guerra et al. 2002 y Gurrutxaga \& Lozano 2008). Por otro lado, una reducción en el tamaño poblacional, con la consecuente pérdida de variabilidad genética provocada por deriva genética y entrecruzamiento, puede volver a las poblaciones más vulnerables a patógenos y enfermedades, aumentando el riesgo de extinción (Lacy 1997; Frankhma 2005; Radwan 2010)

Como un intento de generar información que aporte a la comprensión de este proceso y permita abordar el estudio en mayor profundidad, se eligió el Departamento de Soriano como "caso de estudio". Esta elección se justifica en que Soriano ha sido el departamento sojero por excelencia, ya que concentra alrededor de $40 \%$ de la superficie cultivada en el país (Figura 1). El objetivo general del trabajo fue evaluar los cambios territoriales ocurridos durante el período 19902009 a nivel de paisaje, asociados al proceso de intensificación agrícola.

\section{Materiales y MÉTOdos}

\section{Área de Estudio}

El trabajo se llevo a cabo en el Departamento de Soriano, ubicado entre los $33^{\circ} 03^{\prime} 30^{\prime \prime} \mathrm{S}$ y los $33^{\circ} 53^{\prime} 46^{\prime \prime}$ S; $58^{\circ} 25^{\prime} 32^{\prime \prime}$ O y 57 $13^{\prime} 07^{\prime \prime}$ O. Ocupa una superficie de 833689 ha (Censo General Agropecuario 2000), con suelos caracterizados por su alta fertilidad e importante potencialidad agrícola (MAP / DSF 1976). Según el Censo General Agropecuario del año 2000, 41554 ha (4.98\%) están ocupadas por bosque nativo (BN) y 335098 ha $(40.19 \%)$ por praderas o campo natural $(\mathrm{CN})$. Las actividades productivas predominantes son la agrícola o agrícola-ganadera, seguida de la forestal y lechera, y por último, la hortícola. Desde 1990, el rubro con mayor crecimiento fue la soja, que en la actualidad ocupa $47 \%$ del departamento, seguido por la forestación, con 29000 ha [según el Anuario 2009 (MGAP-DIEA)].

\section{Evaluación del uso del suelo}

Se realizó una evaluación en detalle de los cambios en el uso del suelo, y se analizaron las modificaciones a partir del procesamiento de imágenes satelitales Landsat 5 Themmatic Mapper, mediante el software ArcGis, en dos períodos: 1990 y 2009. Estos años fueron seleccionados porque describen el paisaje de Soriano 10 años antes y 10 años después del 2000, cuando comenzó la expansión acelerada de la actividad sojera en Uruguay (Paruelo et al. 2006). Se utilizaron imágenes que fueron descargadas del sitio del Instituto Nacional de Pesquisas Espaciais de Brasil (www.dgi.inpe.br/CDSR) y correspondían a los meses de verano, cuando los cultivos de soja presentaban un desarrollo vegetal importante, lo que facilita su identificación. Las imágenes de los dos períodos analizados fueron una composición de diferentes fechas debido a que una sola no cubría por entero el Departamento de Soriano. Las fechas fueron 23 de diciembre de 1989 y 15 de enero de 1990, caracterizando el tiempo inicial (1990), y 4 y 13 de febrero de 2009, caracterizando el tiempo final (2009). Cabe aclarar en este caso, que al atravesarse un período de sequía durante el verano 2009, se complementó la clasificación con imágenes del 18 y 27 de octubre de 2009.

La metodología de trabajo que se utilizó para la definición y delimitación de las clases de uso del suelo fue la propuesta en Anno et al. (2003) y Achkar et al. (2004), y se integraron técnicas de teledetección con análisis de información ambiental por medio de un Sistema de Información Geográfica (SIG). Mediante clasificaciones supervisadas y no supervisadas se determinaron las superficies ocupadas por las siguientes categorías de uso del suelo: (1) cultivos, (2) bañados y humedales, (3) zonas urbanas, (4) campo natural), (5) bosques nativos y (6) forestación. La categoría campo natural incluyó a las praderas o campo natural propiamente dicho, campo natural mejorado y zonas que pudieron haber sido agrícolas pero abandonadas y con apariencia de pastizal. En cuanto a la categoría bosque nativo, incluyó principalmente monte ribereño.

Büttner et al. (2004) definen si un objeto será representado como área si sus dimensiones superan $0.2 \mathrm{~mm}$ a la escala del mapa, valor conforme al límite de percepción visual humana para diferenciar objetos a partir de separaciones de $0.2 \mathrm{~mm}$. Dado esto, se recomienda que la unidad mínima cartografiable no sea menor a $4 \mathrm{~mm}^{2}$. Sin embargo en estudios realizados en clasificación de imágenes satelitales para el relevamiento de clases de cobertura del suelo define como unidad mínima cartografiable $25 \mathrm{~mm}^{2}$, valor resultante del compromiso entre el detalle de la información de las clases y del costo que requiere el proceso de clasificación y validación. En este caso, si bien la clasificación se realizó a nivel de píxel de $30 \mathrm{~m} \mathrm{x}$ $30 \mathrm{~m}$ para el análisis de parches se utilizaron solo los mayores a 0.5 ha.

Para validar la clasificación de usos de suelo se realizó una campaña de terreno (febrero de 2009) en la que se relevaron 366 puntos de muestreo, distribuidos al azar dentro del área de estudio. La gran densidad de caminos existentes en el departamento $\left(0.43 \mathrm{~km}\right.$ de caminería $\left./ \mathrm{km}^{2}\right)$ y la ausencia de accidentes geográficos importantes facilitaron el acceso a los puntos seleccionados. La recorrida aleatoria a través de diferentes 
rutas y caminos secundarios del departamento cubrió $\sim 250 \mathrm{~km}$. En cada punto de muestreo se confirmó la posición geográfica y se registró el uso del suelo. La información sobre el uso del suelo registrado a campo en los 366 puntos fue incorporada al SIG; la mitad de ellos fue usada para mejorar la clasificación supervisada y la otra mitad para evaluar la precisión de la clasificación desarrollada. Para la categoría campo natural, la clasificación tuvo un error de omisión de 7\% y de $3 \%$ en bosque nativo, mientras que el error de comisión para campo natural fue $4 \%$ y para bosque nativo $2 \%$.

\section{Evaluación de la diversidad y estructura del paisaje}

Los fragmentos analizados fueron de las categorías campo natural y bosque nativo, ya que, a priori, se consideró que eran suelos más aptos para el uso agrícola que los ocupados por bañados. La evaluación de la estructura del paisaje se realizó en base a descriptores estándar de los fragmentos y del paisaje en su conjunto, mediante la utilización de los softwares ArcGis 9.1 y MS Excel $^{\circledR}$. Los descriptores de los fragmentos fueron: (1) cantidad de fragmentos de las diferentes categorías, (2) superficie, (3) perímetro, (4) índice de forma $\left(\mathrm{F}=\mathrm{P} 2 \pi{ }^{*} \mathrm{~A} \pi\right.$, donde A es el área del fragmento y $\mathrm{P}$ su perímetro) y (5) dimensión fractal ( $\mathrm{FD}=2 \mathrm{ln}$ $\mathrm{P} * \ln \mathrm{A})$, donde $\mathrm{A}$ es el área de cada fragmento $\mathrm{y}$ $\mathrm{P}$ su perímetro).

Los descriptores integrados del paisaje fueron: (6) tamaño medio de fragmento, (7) variabilidad del tamaño de los fragmentos (rango: máximo - mínimo por clase), (8) densidad ( $\mathrm{n}^{\circ}$ parches.100 ha $\left.^{-1}\right)$, (9) fragmentación, (10) conectividad y (11) diversidad. La fragmentación (F) se estimó en base al índice de fragmentación (Gurrutxaga 2003 y Rodríguez Loinaz 2004) dado por la ecuación: $\mathrm{F}=\mathrm{SA} / \mathrm{P} * \mathrm{Rc}$, donde SA es la superficie total del hábitat, $\mathrm{P}$ el número de parches y Rc la dispersión de los parches que se estima como $\operatorname{Rc}=2^{*} \mathrm{dc}^{*}(\lambda / \pi)$. Siendo dc la distancia media desde el centro de un fragmento hasta el fragmento más cercano y $\lambda$ el número de fragmentos por cada 100 ha [(número de fragmentos/superficie total del área de estudio en ha $\left.{ }^{*} 100\right]$. El índice propuesto utiliza una escala inversamente proporcional al grado de fragmentación del paisaje. Un aumento del valor del índice se relaciona con una disminución del grado de fragmentación. Esto es debido a que el incremento de la fragmentación se relaciona con la disminución de la superficie total de fragmentos, un mayor número de fragmentos y una mayor dispersión de éstas (Gurrutxaga San Vicente \& Lozano Valencia 2008).

Para calcular la conectividad se midieron las distancias del centro de un fragmento al borde del fragmento más cercano, para cada uno de los fragmentos (Cayuela 2006 y McGarigal \& Marks 1995). El índice de conectividad se calculó como el promedio de las distancias. La diversidad se calculó mediante el índice de Shannon-Wiener $\left(\mathrm{H}^{\prime}\right)$ a nivel de paisaje (McGarigal \& Marks 1995). Al analizarse al paisaje en su conjunto se consideraron todas las categorías analizadas: cultivos, forestación, campo natural, bosque nativo, bañado y urbano. Para comprobar si existían diferencias estadísticamente significativas en la diversidad encontrada entre los años (1990 respecto a 2009), se compararon los índices de Shannon - Weaver calculados de a pares y mediante una prueba de $t$ (Moreno 2001). También se calculó el índice de equitatividad de Shannon (E) (Pielou 1975 en Badii y Landero 2007). Sus valores oscilan entre 0 y 1 ; aquellos cercanos a 1 indican que las proporciones de cada tipo de categoría son similares, mientras que los cercanos a 0 indican que el paisaje se encuentra dominado por uno o unos pocos tipos de cobertura.

\section{Resultados}

Entre 1990 y 2009, el área cultivada aumentó 27\% (85400 ha) y la forestación pasó de ocupar menos de 2500 a más de 27300 ha (Figura 2). De esta manera se consolidó la zona tradicionalmente agrícola (oeste del departamento) y se produjo una expansión

a) División política. Uruguay (Sudamérica)

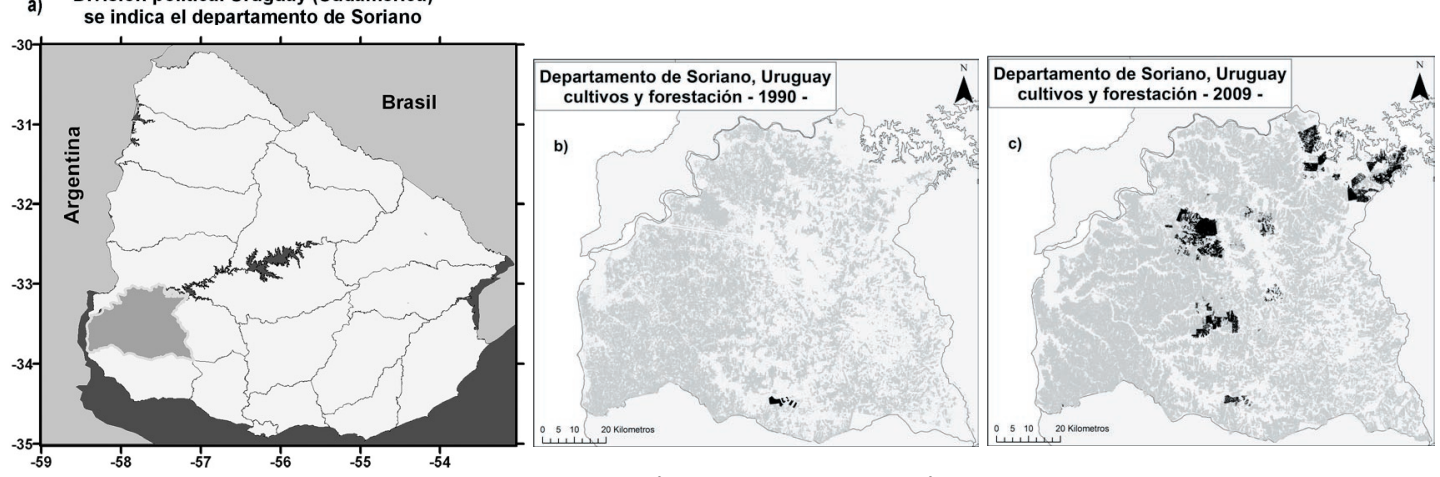

Figura 2. Localización del área de estudio (a) y superficie cultivada (gris) y forestación (negro) en el Departamento de Soriano (Uruguay) según clasificación de uso del suelo realizada en (b) 1990 y (c) 2009.

Figure 2. Study area localization (a) and crop (grey) and forestry (black) area in 1990 (b) and in 2009 (c), according to land use classification. 
Tabla 1. Parámetros evaluados para el año 1990 y 2009 para el Departamento de Soriano, Uruguay, en los dos ambientes considerados $(\mathrm{BN}+\mathrm{CN})$ y juntos como ambientes naturales.

Table 1. Parameters evaluated for 1990 and 2009 for Soriano Department, Uruguay, in the tow environments considered (BN y CN) and both together as "natural environments" (BN+CN).

\begin{tabular}{lcccccc}
\hline & \multicolumn{2}{c}{ Bosque nativo (BN) } & \multicolumn{2}{c}{ Campo natural (CN) } & \multicolumn{2}{c}{$\begin{array}{c}\text { Ambiente natural } \\
\text { (BN+CN) }\end{array}$} \\
\hline & 1990 & 2009 & 1990 & 2009 & 1990 & 2009 \\
$\mathrm{~N}^{\text {o de fragmentos }}$ & 4515 & 2539 & 13.661 & 11430 & 18176 & 13969 \\
Superficie total (ha) & 34004 & 23559 & 412386 & 316531 & 446391 & 340100 \\
Porcentaje del dto. (\%) & 3.8 & 2.7 & 46.6 & 36.2 & 50.5 & 38.9 \\
Perímetro (m) & 6716030 & 5438021 & 34868888 & 43680847 & 41584918 & 49118868 \\
Tamaño promedio (ha) & 7.5 & 9.3 & 30.2 & 27.7 & 24.7 & 24.4 \\
Tamaño máximo (ha) & 1675.6 & 1556.8 & 253713.9 & 234955.6 & 253713.9 & 234955.6 \\
Tamaño mínimo (ha) & 0.5 & 0.5 & 0.5 & 0.5 & 0.5 & 0.5 \\
Densidad (n/100 ha) & 0.54 & 0.29 & 1.64 & 1.31 & 2.05 & 1.60 \\
Índice de forma - promedio & 1.6 & 2.0 & 1.7 & 2.0 & 1.7 & 2.0 \\
Índice de forma - recorrido & $1.07-15.29$ & $1.06-12.71$ & $1.08-66.07$ & $1.03-130.63$ & $1.08-66.07$ & $1.03-130.63$ \\
Índice de fragmentación & 6.34 & 19.78 & 18.27 & 17.38 & 0.09 & 0.12 \\
\hline
\end{tabular}

hacia la zona centro y este. En este período, el bosque nativo pasó de $3.8 \%$ del departamento en 1990 a 2.7\% en 2009; sufrió una reducción de $30 \%$ de su superficie. Por su parte, el campo natural se redujo $23 \%$; pasó de 412000 ha en 1990 a 316000 ha en 2009 (Tabla 1 y Figura 3). Como consecuencia de estos cambios, la diversidad del paisaje sufrió alteraciones significativas, con valores del índice que pasaron de 0.99 en 1990 a 1.02 en 2009 (t calculada $=24.03 ; \alpha=0.05)$. La diversidad máxima potencial obtenida fue de 1.79 (Hmax, calculada como el logaritmo natural del número de categorías analizadas).

La cantidad de fragmentos de bosque nativo pasó de 4515 a 2539; la estructura de tamaños se mantuvo estable (Figura 4a), con una disminución de los fragmentos pequeños mientrasqueelnúmerodefragmentos mayores a 10 ha permaneció igual. En cuanto al tamaño promedio, aumentó de 7.53 ha a 9.58 ha, lo que seguramente se deba a que los fragmentos talados fueron pequeños y dispersos por el territorio y no las grandes masas de bosque nativo presentes principalmente en las márgenes del Río Uruguay y Río Negro. En cuanto al campo natural, de 13661 fragmentos en 1990 , se pasó a 11430 en 2009 , con un tamaño promedio que disminuyó de 30.19 ha a 27.69 ha. La distribución de tamaños de fragmentos no ha variado significativamente, con un número de fragmentos menores a 1 ha y mayores a 100 ha prácticamente constantes, mientras que los intermedios disminuyeron (Figura 4b). En relación a la forma de los fragmentos tanto de bosque nativo como de campo natural, en 1990 los valores muestran una forma más circular, mientras que en 2009 la forma se hace un poco más compleja. De todas maneras, los valores observados de complejidad del borde fueron similares (Tabla 1).

Por su parte, la fragmentación del bosque nativo disminuyó; el índice de fragmentación pasó de 6.3 en 1990 a 19.7 en 2009 (escala inversamente proporcional al grado de fragmentación). La fragmentación del campo natural aumentó de manera leve; el índice varió de 18.3 a 17.3 en 2009 . En términos de conectividad del paisaje, el valor promedio para bosque nativo disminuyó de $365 \mathrm{~m}$ en 1990 (con valores que van desde poco metros hasta $7300 \mathrm{~m}$ ) a $255 \mathrm{~m}$ en 2009 (con valores desde $11 \mathrm{~m}$ hasta $5760 \mathrm{~m}$ ). Para campo natural, en 1990 la distancia promedio entre parches fue de $168 \mathrm{~m}$, con una máxima de $1865 \mathrm{~m}$,

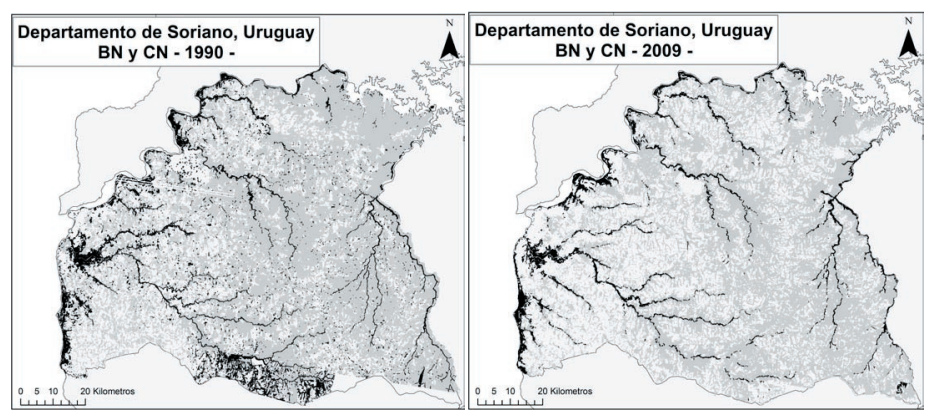

Figura 3. Superficie del Departamento de Soriano (Uruguay) ocupada por bosque nativo $(\mathrm{BN})$ en negro y campo natural $(\mathrm{CN})$ en gris, según clasificación de uso del suelo realizada en 1990 y 2009.

Figure 3. Natural forest $(\mathrm{BN})$ and grassland (CN) area in 1990 and in 2009 according to land use classification of Soriano (Uruguay). BN in black and $\mathrm{CN}$ in grey. 
a)

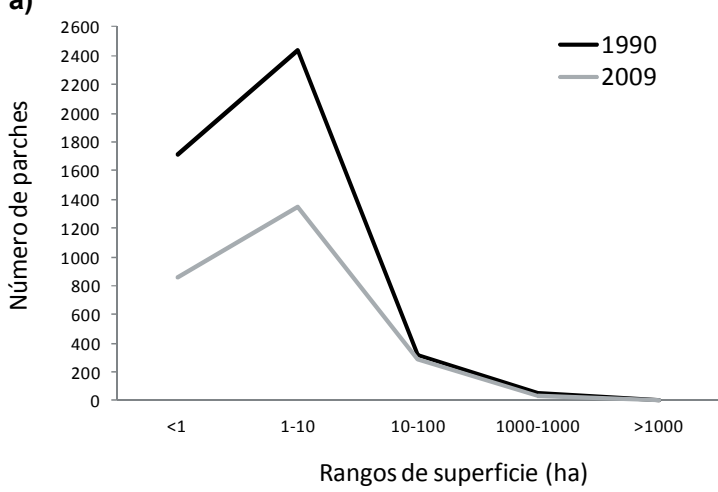

b)

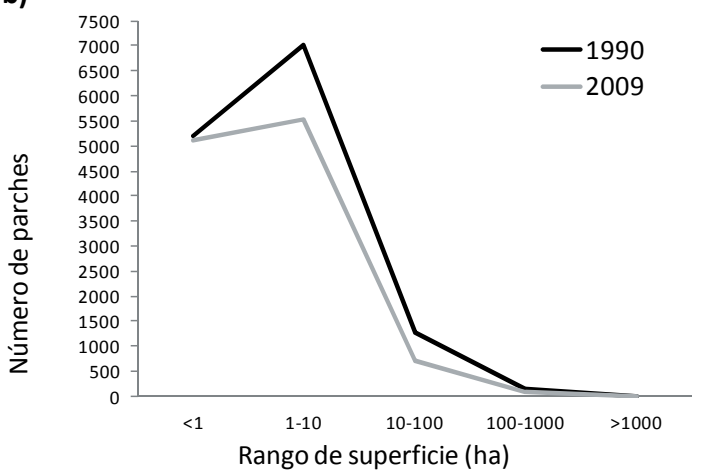

Figura 4. Tamaños de fragmentos de: a) bosque nativo (BN) y b) campo natural (CN) en 1990 y 2009 en el departamento de Soriano (Uruguay).

Figure 4. Fragment sizes of: a) native forest (BN) and b) of grassland (CN) in 1990 and 2009 in Soriano department (Uruguay).

mientras que en 2009 la distancia promedio de separación de los parches aumentó a 192 m, con un valor máximo de $2937 \mathrm{~m}$.

\section{DisCUSIÓN}

Los resultados obtenidos indican que la estructura del paisaje del departamento de Soriano sufrió cambios evidentes durante el período 1990-2009 (Figura 5), asociados a un proceso de intensificación agrícola nacional. Este proceso se caracterizó por una expansión fuerte del cultivo de soja, que pasó, a nivel país, de 8900 ha en $1999-2000$ a 847700 ha en 2009-2010. El área cultivada avanzó sobre campos naturales dedicados a la ganadería extensiva y bosques nativos; se perdieron $23 \%$ y $31 \%$ de estos ecosistemas, respectivamente. Estas diferencias fueron mayores a los errores del métodos de clasificación $(<10 \%)$, lo que determina que los cambios observados son significativos.

En el período estudiado se produjo una disminución del número de fragmentos, tanto de bosque nativo como en el campo natural.
Sin embargo, la diversidad de parches en el paisaje aumentó levemente, pasando de 0.990 en 1990 a 1.020 en 2009. Esto se debió a que la distribución de superficies ocupadas por las categorías de parches se hizo más equitativa, y no por un aumento de la riqueza de parches. La equitatividad pasó de 0.553 en 1990 a 0.569 en 2009 (Máxima equitatividad=1).

Ladisminución delgradodefragmentaciónen bosque nativo se explica por la desaparición de alrededor de 2000 parches boscosos pequeños, convertidos en áreas agrícolas. Estos parches aportaban poco en términos de superficie total, pero originaban una distribución de bosques más extendida y dispersa. Por tanto, su desaparición implicó una distribución más acotada pero más compacta y conectada (i.e., menor distancia promedio entre parches). En cambio, la pérdida de superficie de campo natural estuvo asociada, como era de esperar, a un aumento en la fragmentación y una disminución en la conectividad. La expansión de los cultivos se produjo sobre los suelos con mayor aptitud agrícola, y desplazó a la ganadería hacia el oeste del departamento, donde los suelos son marginales y de mayor fragilidad. Este fenómeno genera riesgos ambientales, especialmente en el caso de los pequeños productores, quienes pueden verse obligados a intensificar el uso del recurso campo natural para subsistir, lo cual degrada la diversidad natural de los pastizales y provoca la erosión de los suelos.

Por otro lado, es importante enfatizar que los bosques nativos en Uruguay están protegidos

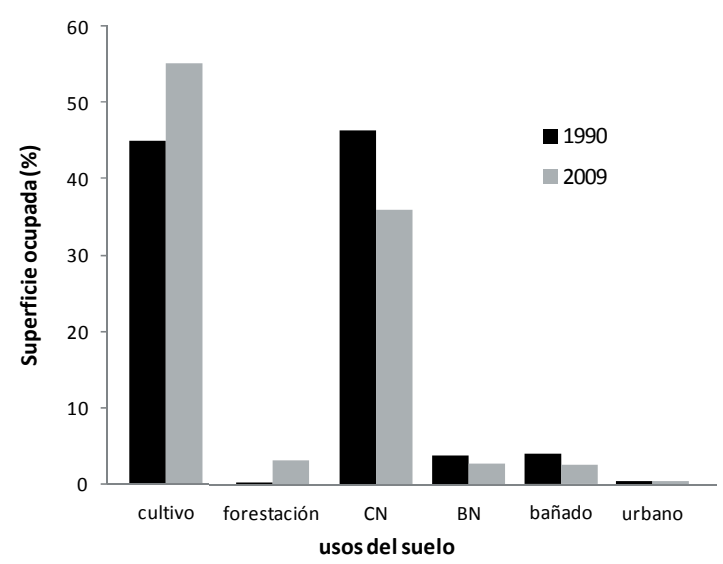

Figura 5. Superficie del departamento de Soriano (Uruguay) ocupada por cada categoría analizada (cultivo, forestación, $\mathrm{CN}=$ campo natural, $\mathrm{BN}=$ bosque nativo, bañado y urbano) en 1990 y 2009.

Figure 5. Area occupied in Soriano (Uruguay) for each analyzed class (crop area, forestry, $\mathrm{CN}=$ grassland, $\mathrm{BN}=$ native forest, wetlands and urban areas) in 1990 and 2009. 
por ley, Decreto No 330/993, que prohíbe la tala indiscriminada y regula el corte y extracción de cualquier producto del bosque indígena. Aún así, en el período estudiado se perdieron en Soriano más de 10000 ha de pequeños parches dispersos de bosque nativo, lo que pone en duda la capacidad estatal para el control y aplicación de la normativa forestal.

La importante disminución de la superficie de los ambientes naturales y alteración de la estructura del paisaje del departamento de Soriano, ocurrida en los últimos 20 años, seguramente tendrá efectos sobre las poblaciones y las comunidades naturales, como así también sobre la capacidad del paisaje de brindarbienes y servicios ecosistémicos. Dentro de los impactos potenciales de estos cambios sobre la biodiversidad, sería particularmente preocupante en el caso de afectar a las especies vulnerables del departamento, como por ejemplo el gato pajero (Leopardus braccatus, Cope 1889) o la mulita (Dasypus hybridus, Desmarest 1804), ambas especies prioritarias para la conservación en Uruguay (Clavijoet al. 2013).

Los escenarios futuros sugieren una profundización del proceso de expansión e intensificación agrícola. El comportamiento de los mercados internacionales provoca la demanda creciente de commodities agrícolas que, junto a la tasa de aumento del consumo mundial, provoca una demanda creciente de productos agrarios; estos procesos continuarán aumentando en el futuro cercano (Brazeiro et al. 2008). Escenarios desarrollados específicamente para Uruguay, a partir de las expectativas gubernamentales de aumento de la producción y la aptitud de suelo para los cultivo de soja, indican incrementos significativos del área sojera para el 2030 (Achkar et al. 2012). En este contexto, la búsqueda de mecanismos que permitan prevenir y mitigar los impactos ambientales negativos dela presente y futura intensificación agrícola, es uno de los principales desafíos ambientales de Uruguay.

\section{BIBLIOGRAFÍA}

Achkar, M; A Blum; L Bartesaghi \& M Ceroni. 2012. Escenarios de cambio de uso del suelo en Uruguay. Informe Técnico, Convenio MGAP/PPR - Facultad de Ciencias / Vida Silvestre/ Sociedad Zoológica del Uruguay/ CIEDUR. Pp. 24.

AchKar, M; D PANario; M Kaemmerer, F AnNo \& V Puech. 2004. Use of SPOT4-5/VEGETATION data for the study of Vegetation Productivity in a humid moderate climate, based on Multivariable Analysis. Bassin A Sánchez - Río Negro
- Uruguay, (1998 - 2002). Second International SPOT/ VEGETATION Users Conference. Antwerp, Belgium.

Anno, F; M Achkar \& V Puech. 2003. Analyse multitemporelle et multirésolution pour l'étude de la productivité végétales en zone climatique tempérée. Bulletin SFPT, 170:30-38.

Badil, MH \& J Landeros. 2007. Cuantificación de la fragmentación del paisaje y su relación con Sustentabilidad. Daena: International Journal of Good Conscience, 2:26-38.

Brazeiro, A; M AchKar, C Toranza \& L Barthesagy. 2008. Potenciales impactos del cambio de uso de suelo sobre la biodiversidad terrestre de Uruguay. Pp. 7-21 en: Volpedo, AV \& LF Reyes (eds.). Efectos de los cambios globales sobre la Biodiversidad. Buenos Aires. CYTED - Programa Iberoamericano de Ciencia y Tecnología para el Desarrollo.

Büttner, G; J Feranec; G Jaffrain; L Mari; G Maucha; et AL. 2004. The CORINE Land Cover 2000 Project. EARSeL eProceedings, 03:331-346.

Cayuela Delgado, L. 2006. Deforestación y fragmentación de bosques tropicales montanos en los Altos de Chiapas, tropicales montanos en los Altos de Chiapas, México. Efectos sobre la diversidad de árboles. Ecosistemas, 15: 192-198.

Censo General Agropecuario. 2000. Censo Agropecuario. Ministerio de Ganadería, Agricultura y Pesca. Dirección de Estadísticas Agropecuarias. Uruguay.

Clavijo, C; JA Martínez-Lanfranco \& A Soutullo(eds.). 2013. Especies prioritarias para la conservación en Uruguay. Vertebrados, moluscos continentales y plantas vasculares. SNAP/DINAMA/MVOTMA y DICYT/MEC, Montevideo. Pp. 222.

Cope, ED. 1889. On the mammalia obtained by the Naturalist Exploring Expedition to southern Brazil. American Naturalist, 23:128-150.

FAHRIG, L. 2001. How much habitat is enough? Biological Conservation, 100:65-74.

Foley, JA; R DeFries; GP Asner; C Barford \& G Bonan. 2005. Global Consequences of Land Use. Science, 309 : 570-574.

FORMANL, RTT. 2005. Land Mosaics: The Ecology of Landscapes and Regions. Cambridge University Press. Pp. 632.

FrankHMa, R. 2005. Genetics and extinction. Biological conservation, 126:131-140.

Green, E; S Cornell; J Scharlemann \& A Balmford. 2005. Farming and the fate of wild nature. Science, 307:550555.

Guerschman, J. 2005. Análisis regional del impacto de los cambios del uso de la tierra sobre el funcionamiento de los ecosistemas de la región pampeana (Argentina). Tesis Doctorado en Ciencias Agropecuarias. Facultad de Agronomía, Universidad de Buenos Aires (Argentina). Pp. 143.

Gurrutxaga San Vicente, M \& P Lozano Valencia. 2008. Ecología del Paisaje. Un marco para el estudio integrado de la dinámica territorial y su incidencia en la vida silvestre. Estudios Geográficos, 69:519-543.

GuRrutxaga SAN Vicente, M. 2003. Índices de fragmentación y conectividad para el indicador de biodiversidad y paisaje de la Comunidad Autónoma del País Vasco. Gobierno Vasco. Dpto. de Ordenación del Territorio. Pp. 32.

Huang, C; S KIm; K Song; JRG Townshend; P Davis; et AL. 2009. Assessment of Paraguay's forest cover change using Landsat observations. Global and Planetary Change, 67:1-12. 
Huang, C; S Kim; A Altstatt; JRG Townshend; P Davis; ET AL. 2007. Rapid Loss of Paraguay's Atlantic Forest and status of protected areas - A Landsat assessment. Remote sensing of Environment, 106:460-466.

LACY, RC. 1997. Importance of genetic variation to the viability of mammalian populations. Journal of Mammalogy, 78:320-335.

MAP/DSF. 1976. Carta de Reconocimiento de Suelos del Uruguay a escala 1/1.000.000. Ministerio de Agricultura y Pesca. Dirección de Suelos y Fertilizantes. Uruguay.

McGarigal, K \& BJ MARKS. 1995. FRAGSTATS: spatial pattern analysis program for quantifying landscape structure. Gen. Tech. Rep. PNW-GTR-351. Portland, OR: U.S. Department of Agriculture, Forest Service, Pacific Northwest Research Station. Pp. 122.

MGAP-DIEA. 1999 al 2009. Anuarios Estadísticos. Ministerio de Ganadería, Agricultura y Pesca. Dirección de Estadísticas Agropecuarias. Uruguay.

Millennium Ecosystem Assessment. 2005. Ecosystems and Human Well-being: Synthesis. Island Press, Washington, DC.

MORENO, CE. 2001. Métodos para medir la biodiversidad. M\&T
- Manuales y Tesis SEA, vol. 1. Zaragosa. Pp. 84.

Múgica de la Guerra, M; JV de Lucio Fernández; C Martínez Alandi; P Sastre Olmos \& JA Atauri-MezQuida. 2002. Integración territorial de espacios naturales protegidos y conectividad ecológica en paisajes mediterraneos. Dirección General de la RENP y Servicios Ambientales Consejería de Medio Ambiente Junta de Andalucía. Pp. 124.

Oyhantçabal, G \& I Narbondo. 2010. Radiografía del agronegocio sojero: El caso de Uruguay. VI Coloquio de la Sociedad Latinoamericana de Economía Política y Pensamiento Crítico (SEPLA), Montevideo.

Paruelo, JM; JP Guerschman; G Piñeiro; EG Jobbágy \& SR VERÓn. 2006. Cambios en el uso de la tierra en Argentina y Uruguay: marcos conceptuales para su análisis. Agrociencia, X:47-61.

Rodríguez Loinaz, G. 2004. Biodiversidad y Paisaje: una estrecha relación. Sustrai, 68:49-52.

RoWANA, J; A BIEDRZYCKA \& W BABIK. 2010. Does reduced MHC diversity decrease viability of vertebrate populations? Biological conservation, 143:537-544.

SCHLESINGER, S. 2008. New wood for old fires. The fever for agrifuels Actionaid. Río de Janeiro, Brasil. Pp. 188. 